\title{
A FAST AND LOW-COST VALIDATION TECHNIQUE FOR ILS AND MLS
}

\author{
P. R. Mahapatra \\ Department of Aerospace Engineering \\ Indian Institute of Science, Bangalore 560012, India \\ M. M. Poulose \\ Telecommunications Research Centre \\ Bangalore 560001, India
}

\begin{abstract}
A feasibility study of evaluating the site effects on ILS and MLS using analytical and computational techniques is presented. The problem of site effect evaluationis divided into three stages: terrain modeling, ray tracing and field evaluation. Innovative techniques are introduced at each stage to make the model versatile enough to handle most general terrain contours and surface conditions and also reduce computational requirements to a minimum. The result is a scheme that can evaluate most terrain types on a mini/microcomputer. A summary of results for actual sites is presented.
\end{abstract}

\section{INTRODUCTION}

Electromagneticsystems for navigationand position location are invariably subject to unwanted "site effects", i.e. interference effects due to rays scattered from various objects within the zone of influence of the systems. Site effects usually introduce errors in position location and navigation, and make the in-situ performance of the equipment less predictable. Among the systems subject to this effect are the Instrument Landing System (ILS) which are operating at a large number of airports around the world to provide landing guidance to approaching aircraft under all weather conditions.

Since aircraft about to land have very low margins of speed and altitude to effect a recovery in case of loss of control, accuracy of guidance information is crucial to safe and comfortable landing. The need for this accuracy is becoming even higher as air traffic densities are increasing at terminal areas and there is pressure to increase the landing rates. Inaccurate landing guidance provided by the ILS has been implicated in many air disasters, and a need has been felt to improve the accuracy of the ILS and reduce its vulnerability to site effects.

The Microwave Landing System (MLS) is a response to the need for greater traffic handling capacity, accuracy and versatility of landing guidance and reduced susceptibility to disturbing effects of site reflections. However, the MLS, which has gone through an extended period of development and proving and is at the beginning phase of operational installation, has the possibility of being affected by certain kinds of interference, especially because of the dense traffic that the system is expected to handle.

The ILS is currently the principal landing aid. According to projections, the MLS will have been installed at a sufficient number of important airports around the world during the next decade or two to become the predominant landing system. However, till then the ILS will remain the main landing aid, and even after the MLS has established itself, the ILS will continue to be a major landing aid, serving the relatively low-traffic airports which cannot justify the investments on MLS and airports in the less developed parts of the world.

In any installation of the ILS or the MLS, it is important to keep the site effects within limits to ensure that the equipment performs as intended. Currently, site evaluation for the ILS (MLS is not yet in wide use) is routinely done experimentally. For initial site evaluation prior to actual installation of an ILS, temporary equipment of specifications similar to the proposed equipment are installed at the designated site and the quality of the resulting glideslope is evaluated by airborne measurements conducted according to International Civil Aviation Organization 


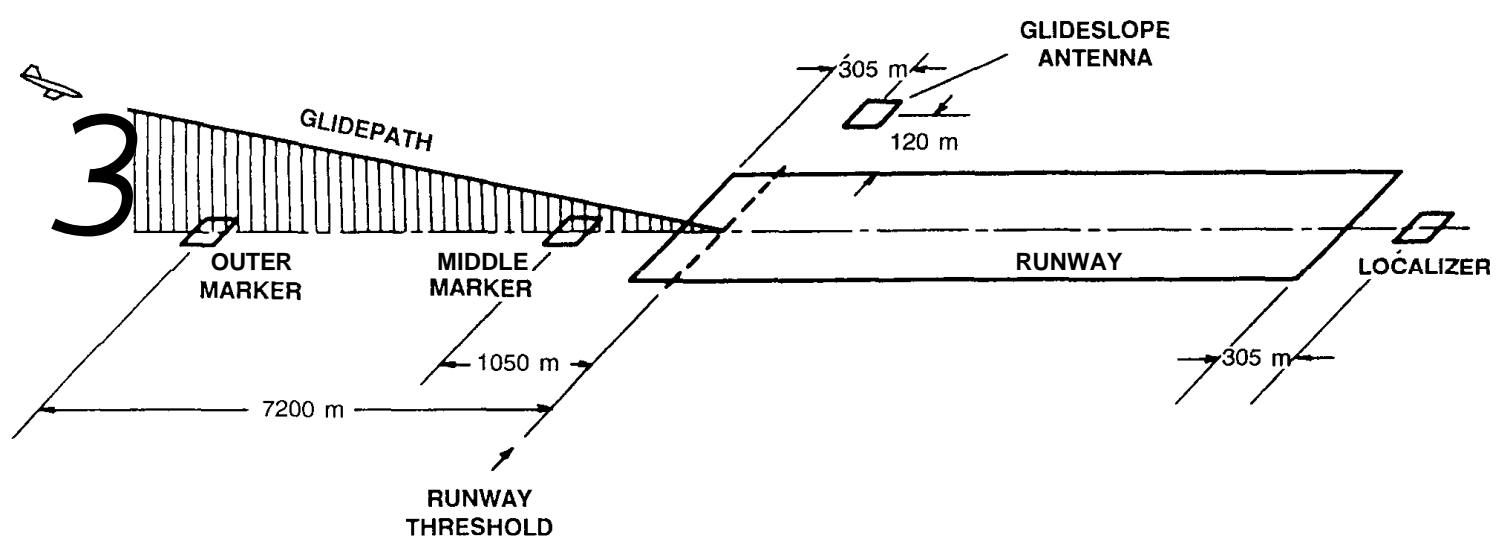

Figure 1. Typical ILS layout

(ICAO) guidelines. Such a procedure is invariably time consuming, requiring several months, and is expensive, requiring hundreds of thousands of dollars. Even after an ILS has been installed and operating, such experimental evaluation of glideslopes are to be conducted periodically to ensure that the system performance stays within limits in spite of changes in the site and the equipment. There is thus a strong need to develop analytical models of site effects that are accurate enough to replace experimental procedures to a large degree. Site effects could then be evaluated computationally, which would require far less time and expense, given the low cost and high accessibility of modern computing systems. This paper provides the outlines of such a method.

\section{SYSTEM DESCRIPTION}

The ILS has been extensively described in literature $[1,2]$, as it has been in service now for about four decades. It has three major subsystems which provide landing guidance to approaching aircraft in three dimensions. The subsystems are: 1. Glideslope, 2. Localizer and 3. Marker Beacons. Their arrangement with respect to a runway is shown in Figure 1.

The glideslope equipment has a carrier frequency in the $328-336 \mathrm{MHz}$ band. It provides vertical guidance (i.e. fly-up/down information) to approaching aircraft with respect to a designated glideslope plane. The glideslope equipment generates two shaped beams carrying 90 and $150 \mathrm{~Hz}$ modulation, respectively, and the vertical guidance information is obtained at the aircraft's current location by sensing the differential depth-of-modulation (DDM) between the signals received via the two beams. The DDM is expected to be zero ("nulled") along the designated glideslope plane, and to vary linearly over an angular sector on either side of the plane.

The localizer subsystem operates in the 108-112 $\mathrm{MHZ}$ band and provides lateral guidance (fly-left/right) information to approaching aircraft with respect to a vertical plane through the runway center line. The localizer operation is also based on the null-DDM principle, the nulling being expected to occur along the extended center line of the runway. Like the glideslope, the localizer also has a sector of linear DDM variation on either side of the null-DDM plane.

The intersection of the nominal glideslope plane and the vertical plane through the runway center line is the nominal glidepath line on which both the DDMs (those of the glideslope and the localizer) are expected to be zero. The pilot of the landing aircraft attempts to fly the glidepath down to the final landing operation by simply holding the aircraft in such a course that keeps both the glideslope DDM and the localizer DDM individually at zero.

The practical situation may be quite different, however, from the idealized operation stated above. Because of a variety of factors, including (most importantly) site effects, the DDMs usually are not exactly zero on the nominal planes on which they are expected to null. Conversely, the surfaces of nullDDMs are actually warped versions of the nominal 


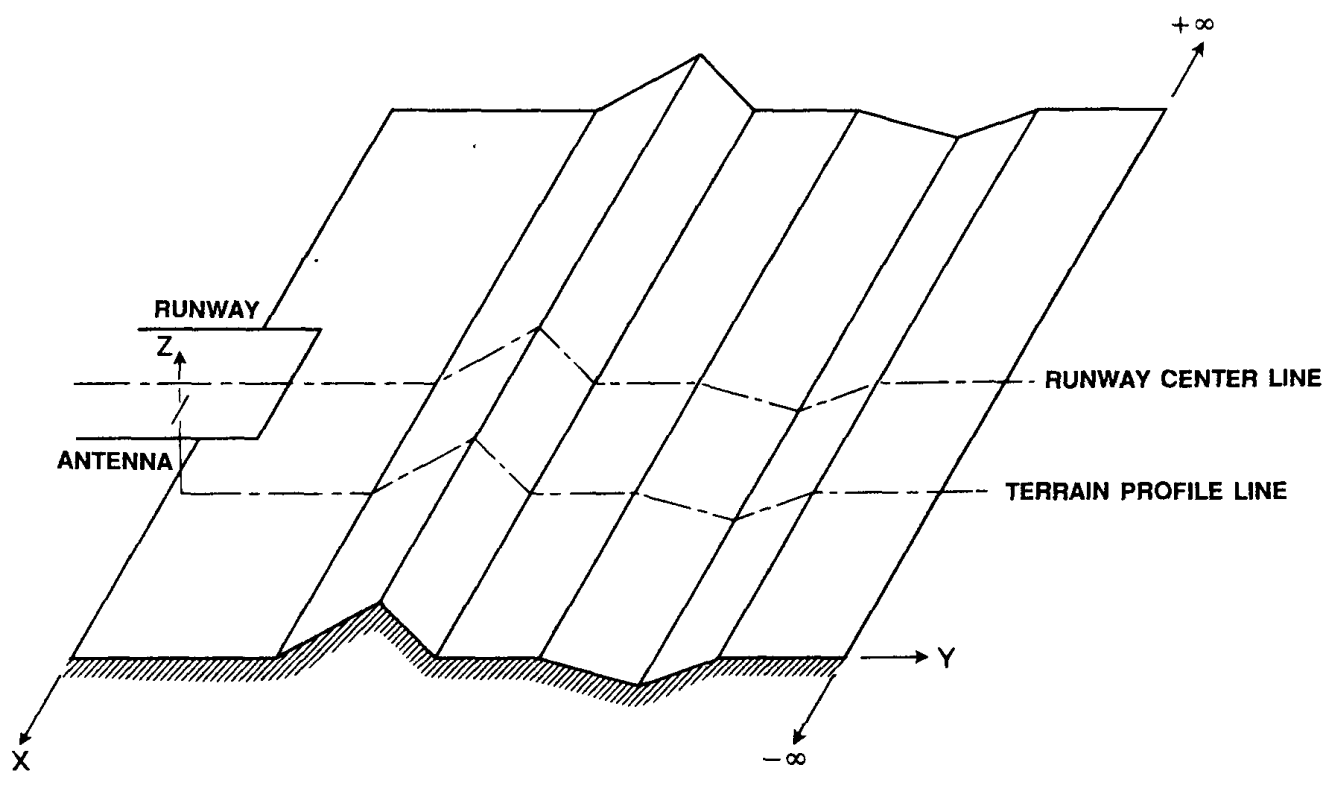

Figure 2. Multiplate or multiwedge model of terrain

planes. Thus, when the aircraft attempts to fly a nullDDM course, it actually encounters kinks and bends on the glidepath line, which is the intersection of the two warped planes. Flying such a course results in rough rides. If the amplitudes of these glidepath undulations are large, the aircraft may lose control in trying to negotiate them. In extreme cases, the apparent glidepath may intersect terrain features or other obstructions, resulting in direct crash of the aircraft.

The third subsystem of the ILS, the marker beacons, are located at a few points (usually two or three) along the aircraft approach path. Operating at $75 \mathrm{MHz}$, they provide discrete pin-point range fixes as the aircraft flies through their fixed fan-shaped beams during landing. Since the fan beams of the $75-\mathrm{MHz}$ marker beacons point vertically upward, with very little power radiated horizontally, these are not subject to significant site effects.

Between the glideslope and the localizer, the former is more susceptible to site effects. This is because ground undulations which are the primary cause of site effects in the case of ILS, have an essentially horizontal orientation, and scatter relatively small amount of energy in the principal direction (horizontal, i.e. left/right) of localizer guidance. Because of this, and also because errors in the vertical direction are more critical for obstacle clearance and touchdown, we confine attention to the glideslope alone in the context of site effects.

The MLS [3] has a more elaborate design which has the objectives of increasing the traffic handling capacity and reducing site effects. Both these advantages are inherent in the currently accepted configuration of MLS -- the Time-Referenced Scanning Beam (TRSB) MLS. The higher operating frequency of MLS in the $5 \mathrm{GHz}$ band permits a narrower beam than the ILS. The MLS utilizes one oscillating or "nodding" beam in each planes of guidance (vertical and horizontal), unlike the two static or "staring" beams of the ILS. An approaching aircraft's position information is available to the aircraft itself in the form of the time difference between two pulses received by its antenna that correspond to the passage of the nodding beam by the aircraft in its upward and downward (or left and right) strokes. The nodding configuration makes it possible for the MLS to cover a much larger sector than the ILS with a narrower beam. Further, extensive effort has been put into the design of the radiation patterns of the MLS antennas to achieve a sharp cutoff at ground-grazing angles. This minimizes the power radiated in the direction of terrain features, resulting in 
reduced site effects.

There are, however, reasons that the study of site effects is important for the MLS. First, the standard terrain conditions assumed for the MLS may not be valid everywhere. For example, although the lower scanning limit of $0.9^{\circ}$ for the vertical scan ensures that the elevation coverage beam stays clear of level ground at its lowest position, this may not be so if the ground has significant slope, as is the case over extended regions in many parts of the world. Further, scattering due to sidelobes could have significant interfering effect.

Next, the effects of interference on MLS may be more critical than the ILS. This is because the MLS is designed to provide multiple approach paths to aircraft. There is thus less margin for error in the MLS than the ILS in which errors become catastrophic only when they are so large as to affect obstruction clearance. A second reason for the sensitivity of the MLS is its higher operating frequency which would make smaller scatterers appear electrically larger (i.e. large in terms of wavelength). Thus, the effect of objects like parked or moving automobiles or aircraft, which are not significant at ILS frequencies, will be enhanced at the MLS frequencies.

\section{TERRAIN TYPES AND MODELING}

To study the effects of interference due to electromagnetic energy scattered by the terrain, it is necessary to model the characteristics of the terrain responsible for scattering. In the context of the ILS, nearly all the scattering effect is confined to a narrow strip of ground along the ground projection of the aircraft approach path, extending a few kilometers ahead of the runway.

In modeling the terrain, both the shape as well as the electromagnetic characteristics of the surface need to be taken into account. The shape should ideally be described as a bivariate function which exactly specifies the height of each point on the terrain in terms of its horizontal $(x-y)$ coordinates. However, such a description would make scattering computations difficult, and hence a simpler description is desirable.

One simple and widely used model for terrain effect studies is the "multiplate" or "multiwedge" model. In such a model (see Figure 2) the terrain is assumed to consist of a succession of flat plates. Since adjacent pairs of plates may be considered as constituting wedges, the model may also be viewed as consisting of a succession of straight-edged wedges. The model is so formed that the edges of the wedges are perpendicular to the extended runway center line.

Although the multiplate model is rather simple, it is quite valid (indeed accurate) for application to ILS, since only a narrow but long strip of ground is involved in scattering. Because of the small width (of the order of a couple of hundred meters) of the strip, variations of terrain height across the strip can be neglected, resulting in the one-dimensional variation modeled by the multiplate shape.

The generation of a multiplate or multiwedge model for a particular airport is quite straightforward. The starting point is the contour map of the airport and its surrounding areas, which is normally readily available. Taking a section of this contour map along a line from the glideslope antenna parallel to the runway center line gives the terrain profile line (Figure 2). The profile line is then approximated by straight line segments. For mild undulations, such as are found commonly at airport sites, less than 10 straight line segments have been found to be adequate, but a larger number may be necessary for more undulating sites found in hilly areas. After the straight-segmented profile line is formed, it is translated laterally parallel to itself to generate the multiwedge model of the terrain.

Other terrain surface properties that affect its scattering behavior are the impedance and roughness of the surface. In a majority of locations the terrain surface impedance may be neglected, i.e. the surface assumed to be fully conducting. This is because normal soil with significant loam and moisture content has low surface impedance which has negligible influence on the its scattering properties at the very low incidence angles of radiation encountered in the ILS. However, in certain special locations. especially those involving dry and sandy soils, impedance effects cannot be neglected and must be built into the model.

The roughness of the terrain surface is also an important parameter in determining its scattering characteristics. However, it is possible to incorporate roughness effects into the model by using an equivalence relation which expresses the roughness in terms of an equivalent surface impedance which may be added to the inherent surface impedance. The multiwedge approximation, after incorporation of the 
surface impedance and roughness effects, constitutes the complete model of the terrain that is used to evaluate the site effects.

\section{ILS PERFORMANCE EVALUATION}

Using the model described above it is possible to proceed with the evaluation of the quality of the ILS glideslope at a particular site. This is done by using a ray-theoretic approach for the evaluation of electromagnetic fields. Since the terrain features responsible for site effects are large compared with the operating wavelength of the ILS (this is even more true for MLS), a high-frequency scattering assumption is valid. This permits a ray-theoretic approach.

Evaluation of the electromagnetic fields due to each antenna element at a given instantaneous location of the approaching aircraft involves the following two major steps:

1. Ray Tracing, which involves the determination of all the possible ray paths from each antenna element to the current position of the aircraft. In addition to the direct (D) ray from each glideslope antenna element to the aircraft, numerous rays will reach the aircraft after being scattered by the ground. A ray striking the terrain, modeled as multiple plates or wedges, may suffer reflection on a plate surface or diffraction at an edge. Rays emanating from an antenna element may reach the aircraft after either a single reflection or diffraction or a sequence of reflections and/or diffractions in any order. Accordingly, rays may be classified as singly reflected (R) or diffracted (Df) rays, or higher order rays such as reflected-reflected (RR), reflected-diffracted (RDf), diffracted-reflected-diffracted (DfRDf), etc.

The order of a ray is the number of reflections and/or diffractions a beam undergoes over the model terrain before reaching the aircraft. The orders of rays in case of a multiwedge terrain model will in general be infinite. However, the contribution of rays to glideslope aberration will generally diminish with increase in their order. We have determined after repeated evaluations that rays beyond the third order have negligible influence on glideslope performance.

Although all sequential combinations of reflection and refraction within any given order group are possible, not all such combinations may exist, for a given model terrain, between a given antenna element and an instantaneous location of the aircraft. The existence of each ray combination must therefore be meticulously checked before its contribution to electromagnetic interference is to be evaluated. This can be highly cumbersome, given the very large number of rays. Since the point of reflection of each ray on any given plate of the terrain model is not known a priori, the reflection points may be determined through a numerical search, as has often been done in the past. But the computational burden for such search would increase exponentially with the order of the ray, forcing the ray-tracing to be confined to only the lowest few orders. We have developed an allgeometric ray-tracing scheme, and the necessary programs, to exhaustively trace and determine the existence of each possible ray up to any specified order.

2. Field Evaluation consists of determining the electromagnetic fields due to each existing ray at the aircraft location, and summing up all the complex field contributions to obtain the resultant field. The primary contribution comes from the direct ray from the antenna element to the aircraft. To evaluate the contribution due to terrain-scattered rays responsible for the interference, the scattering process must be modeled appropriately. In the high-frequency approach, the reflection process is readily modeled by using simple classical rules. However, the understanding of the diffraction process has been evolving in the recent decades.

Of the current formulations for the evaluation of diffracted fields, two "uniform" theories are best known: the Uniform Theory of Diffraction (UTD) [4] and the Uniform Asymptotic Theory (UAT) [5]. These theories assume the scatterer to be perfectly conducting, and provide continuous and bounded field estimates around the scatterers. We have used both these theories in cases where the terrain type permits the use of the perfect-conductivity assumption.

For the high-impedance terrain types, e.g. dry.sandy terrain as mentioned earlier, for which the perfect-conductivity assumption would be too far from reality, we have extended the original UTD formulation to include impedance effects [6]. Using this theory, the terrain-scattered electromagnetic fields for airports with relatively high-surface-impedance terrain and/or roughness have been evaluated. 


\section{DEDUCTION OF GLIDESLOPE QUALITY}

Since the glideslope is established electronically by a nulling of the DDM, it is necessary to compute the DDM from the field contributions due to the rays at the aircraft location. As a first step, the combined contribution due to all the rays reaching the aircraft from each antenna subarray is computed separately. Depending on the antenna configuration, there are typically two or three antenna subarrays mounted on a common mast. Once these field components are available, the DDM can be readily determined for each antenna configuration using a simple algebraic relation.

Using this method of DDM evaluation at individual points in space, the DDM variation is obtained along designated aircraft flight paths. Two specific flight paths are stipulated by ICAO for glideslope evaluation purposes [7]:

1. The Level Run determines the vertical characteristic of the glideslope. In this run, DDM values are determined along a constant-altitude flight path, typically at $100 \mathrm{ft}(305 \mathrm{~m})$ altitude in a vertical plane passing through the nominal glidepath. From the variation of the DDM with the elevation angle, the true glideslope angle and the terrain sector limits are determined as those elevation angles at which the DDM has values of zero and r75 microamperes, respectively.

2. The Low-Level Approach is used to measure the structural characteristic of the glideslope. This flight path is along the nominal glideslope. The DDM, which should ideally be zero at all points on this path, will normally show some residual values due to various imperfections. The glideslope is declared to be normal if these aberrations stay within specified limits.

In actual practice these runs are conducted by flying instrumented aircraft along the two flight paths and recording the measured DDM along the flight path. In our exercise, we have used the same flight paths, but have computed the DDM at closely spaced discrete points along the paths using analytical field evaluation techniques.

The method has been tried using terrain data at a few actual airport sites equipped with ILS. The results of actual flight tests are available for these airports. The analytical results have been compared with test flight results to validate the analysis.
The results have shown that under the perfectconductivity assumption, UTD and UAT provide quite comparable results in terms of accuracy, with the latter having a slight edge in accuracy in certain cases. UAT, however, requires a significantly higher level of computation than UTD. The magnitudes of errors in glidepath angle computation remains within $0.02^{\circ}$, and those in sector width computation remain within about 0.04 ".

For terrain with high surface impedance and roughness, we have used our modified UTD incorporating surface impedance effects. The results show a definite improvement over the application of the original UTD and UAT. For an airport with terrain undulations somewhat above $\pm 1 \mathrm{~m}$ with respect to the horizontal surface through base of the antenna mast, dry sandy terrain and moderate surface roughness, the extended UTD predicts the glidepath angle correctly to the second decimal place and the sector width within an accuracy of $\pm 0.01^{\circ}$.

\section{CONCLUDING REMARKS}

The feasibility and validity of an analytical approach to the determination of the quality of ILS performance in the presence of non-ideal terrain has been dealt with in this paper. It has been shown that using a simple 2-dimensional multiwedge model of the terrain (i.e. there is no variation of terrain height in the third dimension), it is possible to obtain accurate estimates of the glideslope parameters by simulating procedures similar to those specified by ICAO., The computational results have been validated by comparison with actual data from flight tests.

The choice of a simple model and a closed form geometric method for ray tracing has drastically cut down computational times, making it possible for the evaluation program to run on minicomputers and even on micro/personal computers. For terrain types for which the perfect-conductivity assumption is valid, results for about a hundred points along a test flight path can be obtained with a few tens of minutes of computation on a modern minicomputer, depending on the number of plates/wedges in the model. The incorporation of impedance into the model increases the computational needs several fold, requiring upto a few hours for the same run. However, with the widespread availability and low cost of powerful miniand microcomputers, the cost and time for such 
computation is negligible in comparison with experimental procedures. Such fast and inexpensive evaluation will permit many re-runs for a given site with various possible site developments or modifications to determine the level of development that would make a bad site acceptable for ILS/MLS installation.

Actual site evaluations have been summarized in the paper only for the ILS, since experimental data for MLS site evaluation have not been generally available. However, the methodology is expected to work for the MLS as well, with the use of appropriate system parameters.

The outline and results presented here are to be considered only as a feasibility study. Clearly, much more validation including diverse equipment and terrain types in different geographical regions of the world would be necessary before the method can be recommended to replace the current experimental procedure $s$ with confidence. Nevertheless, the success of the current attempt is a significant step in the right direction.

\section{ACKNOWLEDGEMENT}

During the writing of this paper, the authors have been Research Associates of the National Research Council.

\section{REFERENCES}

[1] Kayton, M., "Landing guidance," in Avionics Navigation Systems, Eds.: M. Kayton and W. R. Fried, Ch. 14. New York: John Wiley, 1969.

[2] Plessey Radar: Technical Manual on ILS, TP 3140 , Addlestone, England, 1972.

[3] Poulose, M. M., P. R. Mahapatra and N. Balakrishnan, "Microwave landing system -- a favored alternative to the current ILS," IFTE Tech Review, Vol. 2, 1985, pp. 375-382.

[4] Kouyoumjian, R. G. and P. H. Pathak, "A uniform geometrical theory of diffraction for an edge in a perfect conducting surface," Proc. IEEE, Vol. 62, 1974, pp. 1448-1461.

[5] Ahluwalia, D. S., R. M. Lewis and J. Boersma, "Uniform asymptotic theory of diffraction by a plane screen," SIAM J. Appl. Math., Vol. 16, 1968, pp. 783-807.

[6] Poulose, M. M. and P. R. Mahapatra, "ILS glideslope evaluation for imperfect terrain," IEEE Trans. on Aerospace and Electronic Systems, Vol. AES-24, 1988, pp. 186-191.

[7] International Civil Aviation Organization: Manual on Testing Radio Navigation Aids, Annexure II. Montreal, Canada: ICAO. 